\title{
Calf massager: intervention for body muscle discomfort during prolonged standing
}

\begin{abstract}
Many occupations require workers to stand for long periods of time without proper interventions, which causes discomfort in the back and lower limbs. Therefore, this study aims to assess the effectiveness in alleviating body muscle discomfort during prolonged standing through the use of a calf massager. This study was conducted among male workers at a manufacturer with production line workers and the list was obtained from the HR Department and simple random sampling was done by number categorization. A total of 100 respondents (50 respondents for both the control and the experimental groups) participated in this study. The experiment took place in a room with a similar setup for the production line. Each respondent was requested to perform the simulated task for $2 \mathrm{hr}$. For the experimental group, the calf massager was turned on every $15 \mathrm{~min}$. At every 15-min interval after turning on the calf massager, respondents from both groups were required to evaluate their discomfort level on a Borg's scale CR-10 questionnaire. The results showed that the level of body discomfort among respondents in the experimental group reduced (20-30\%) compared with that of the control group. Multivariate analysis results revealed that the discomfort rating for the lower back, knees, thighs, calves, and feet was significantly lower $(\mathrm{p}<.05)$ among the experimental group compared with the control group. For lower body parts, the lower back region was statistically significant $(\mathrm{p}<.05)$ at the 90th, 105th, and 120th min; the thigh region was statistically significant $(\mathrm{p}<.05)$ at the 120 th min; the knee region was statistically significant $(\mathrm{p}<.05)$ at the 105 th and 120 th $\mathrm{min}$; the calf region was statistically significant $(\mathrm{p}$ $<.05)$ at all minute intervals except the 15 th and 45th $\mathrm{min}$, while the feet region, was statistically significant at the 105th and 120th min. Therefore, this study indicates that calf massage treatment is capable of reducing body muscle discomfort during prolonged standing and highlights the significance of calf massage.
\end{abstract}

Keyword: Body discomfort; Borg scale; Calf massage; Discomforting rating; Prolong standing 